\title{
RESISTÊNCIA À COMPACTAÇÃO DE UM LATOSSOLO CULTIVADO COM CAFEEIRO, SOB DIFERENTES SISTEMAS DE MANEJO DE PLANTAS INVASORAS ${ }^{(1)}$
}

\author{
Cezar Francisco Araujo-Junior ${ }^{(2)}$, Moacir de Souza Dias Junior ${ }^{(3)}$, \\ Paulo Tácito Gontijo Guimarães ${ }^{(4)} \&$ Bruno Silva Pires ${ }^{(5)}$
}

\begin{abstract}
RESUMO
O conhecimento dos níveis de pressão que podem ser aplicados aos solos submetidos a diferentes sistemas de manejo das plantas invasoras é essencial para adaptar o manejo de lavouras cafeeiras de forma sustentável. O objetivo deste estudo foi o de avaliar a influência dos diferentes sistemas de manejo de plantas invasoras sobre a resistência à compactação em três camadas de um Latossolo Vermelho-Amarelo (LVA), utilizando modelos de capacidade de suporte de carga (CSC). O estudo foi realizado na fazenda da Epamig, no município de PatrocínioMG, em uma lavoura cafeeira implantada em fevereiro de 1999, com a cultivar Rubi 1192 no espaçamento de $3,8 \times 0,7 \mathrm{~m}$. Foram avaliados os efeitos de quatro sistemas de manejo de plantas invasoras: sem capina (SC); capina manual (CM); herbicida de pós-emergência (HPÓS) (glyphosate); e herbicida de pré-emergência (HPRÉ) (oxyfluofen). Em cada sistema de manejo, foram coletadas 15 amostras indeformadas, em julho de 2004, no centro das entrelinhas, nas camadas de 0-3, 10-13 e 25-28 cm, totalizando 180 amostras. As amostras indeformadas foram equilibradas em diferentes umidades e submetidas ao ensaio de compressão uniaxial, para obtenção dos modelos de CSC. Os resultados sugerem que, de maneira geral, a resistência à compactação avaliada através da CSC decresce no centro da entrelinha na seguinte ordem: HPRÉ na camada de
\end{abstract}

${ }^{(1)}$ Parte da Tese de Mestrado do primeiro autor apresentada ao Programa de Pós-Graduação em Agronomia/Solos e Nutrição de Plantas do Departamento de Ciência do Solo da Universidade Federal de Lavras - DCS/UFLA. Projeto financiado pelo Consórcio Brasileiro de Pesquisa e Desenvolvimento do Café CBP\&D/Café. Trabalho apresentado no XXXI Congresso Brasileiro de Pesquisas Cafeeiras, Guarapari-ES. 2005. Recebido para publicação em janeiro de 2007 e aprovado em setembro de 2007.

(2) Engenheiro-Agrônomo, Mestre em Solos e Nutrição de Plantas. Doutorando em Ciência do Solo do Departamento de Ciência do Solo da Universidade Federal de Lavras - DCS/UFLA. Caixa Postal 3037, CEP 37200-000 Lavras (MG). Bolsista do CNPq. E-mail: cfaj@bol.com.br

${ }^{(3)}$ Engenheiro-Agrícola, PhD em Crop and Soil Science. Professor do Departamento de Ciência do Solo, UFLA. Bolsista CNPq. Email: msouzadj@ufla.br

(4) Pesquisador da Empresa de Pesquisa Agropecuária de Minas Gerais - EPAMIG. Campus UFLA, Caixa Postal 176, CEP 37200000 Lavras (MG). E-mail: paulotgg@epamig.br

${ }^{(5)}$ Engenheiro-Agrônomo, Mestre, Doutorando em Ciência do Solo do Departamento de Ciência do Solo, UFLA. Professor da FESP-UEMG Campus de Passos-MG. Bolsista do CNPq. E-mail: piresbruno@passosuemg.br 
0-3 cm > CM na camada de 10-13 cm > SC nas camadas de 0-3, 10-13 e 25$28 \mathrm{~cm}=$ HPÓS nas camadas de 0-3, 10-13 e 25-28 $\mathrm{cm}=$ CM nas camadas de 0-3 e 25-28 cm = HPRÉ na camada de 10-13 cm > HPRÉ na camada de $25-28 \mathrm{~cm}$. A camada que se mostrou mais suscetível à compactação foi a de 25-28 cm, do sistema de manejo HPRÉ, e a mais resistente foi a camada de 0-3 cm do sistema de manejo HPRÉ. Os sistemas de manejo SC e HPÓS nas camadas de 0-3, 10-13 e 25-28 cm, CM nas camadas de 0-3 e 25-28 cm e HPRÉ na camada de 10-13 cm apresentaram a mesma resistência à compactação.

Termos de indexação: pressão de preconsolidação, sustentabilidade, modelos de capacidade de suporte de carga, suscetibilidade, Coffea arabica $\mathrm{L}$.

\section{SUMMARY: RESISTANCE TO SOIL COMPACTION OF AN OXISOL CULTIVATED WITH COFFEE PLANTS UNDER DIFFERENT WEED MANAGEMENT SYSTEMS}

The knowledge of the pressure levels that can be applied to the soil under different weed management system (WMS) is importante for coffee plantations management. The objective of this study was to evaluate the influence of different weed management systems on the susceptibility to compaction of a Red-Yellow Latossol (Oxisol) (LVA) using soil support capacity (CSC) models. This study was carried out at the Epamig Research Farm in Patrocínio, state of Minas Gerais, Brazil, in a coffee plantation using Ruby 1192 coffee variety in a $3.8 \times 0.7 \mathrm{~m}$ spacing, planted in February 1999. Four WMS were used and the soil samples were collected in-between the rows under the following management systems: (1) no weed control (SC); (2) hoe-weeded (CM); (3) weed control with post-emergence herbicide Glyphosate (HPÓS); (4) weed control with pre-emergence Oxyfluorfen (HPRÉ). Fifteen undisturbed soil samples from each system were collected (in the layers 0-3, 10-13 and $25-28 \mathrm{~cm}$ ) in July 2004, totaling 180 samples. The undisturbed soil samples were equilibrated at different moisture contents and subjected to the uniaxial compression test to obtain the soil CSC models. Results suggested that the support capacity of the LVA decreases in the center of the inter rows in the following order: HPRE in the $0-3 \mathrm{~cm}$ layer $>$ $C M$ in the 10-13 cm layer $>S C$ in the $0-3,10-13,25-28 \mathrm{~cm}$ layers = HPÓS in the 0-3, 10-13, 25-28 cm layers $=C M$ at 0-3 and 25-28 cm layers $=H P R E$ in the 10-13 cm layer $>$ HPRE in the 25-28 cm layer. Weed control with HPRE in the 25-28 cm layer was most susceptible, while HPRÉ in the $0-3 \mathrm{~cm}$ layer was most resistant to soil compaction. The management systems $S C$ and HPÓS in the 0-3, 10-13, 25-28 cm layers and the managements CM in the 0-3 and 25-28 cm layers and HPRE in the 10-13 cm layer were equally susceptible to soil compaction.

Index terms: preconsolidation pressure, sustainability, support capacity models, susceptibility, Coffea arabica $L$.

\section{INTRODUÇÃO}

O café é o segundo produto mais comercializado no mundo, ficando atrás apenas do petróleo. O Brasil lidera há dois séculos a produção mundial de café, e sua história política, econômica e social sempre esteve vinculada a ciclos de preços do produto. A produção do País corresponde, em média, a $33 \%$ da produção mundial, com produção na safra de 2005/2006 em torno de 33,3 milhões de sacas de café. Minas Gerais é o maior produtor nacional, com produção em torno de 15,6 milhões de sacas, que corresponde a, aproximadamente, $47 \%$ da produção nacional (Agrianual, 2006). Entretanto, para maior competitividade da cafeicultura nacional, é necessário se enquadrar às exigências impostas por consumidores internacionais.

Dentre as exigências a serem cumpridas pelos cafeicultores, destacam-se critérios socioambientais e métodos que considerem a conservação da biodiversidade e suas condições de sobrevivência para promover um manejo sustentável, proporcionando melhoria ou manutenção da sua estrutura do solo, de forma a evitar sua compactação pelo manejo inadequado (BSCA, 2005). A compactação do solo tem sido identificada como um dos principais processos causadores de degradação da sua estrutura, comprometendo a qualidade física de terras agrícolas 
e a obtenção de maior produtividade (Canillas \& Salokhe, 2002; Alakukku et al., 2003; Peng et al., 2004; Dias Junior et al., 2005). Esse tipo de degradação ocorre devido ao aumento na intensidade de tráfego e da carga aplicada (Kondo \& Dias Junior, 1999; Silva et al., 2003; Dias Junior et al., 2007).

Os diferentes sistemas de manejo de plantas invasoras utilizados em lavouras cafeeiras podem alterar atributos químicos, físico-hídricos e biológicos do solo, alterando o comportamento compressivo e, conseqüentemente, a capacidade de suporte de carga do solo. Assim, o manejo de plantas invasoras não pode ser analisado somente a partir de observações pontuais de um processo de competição por água e luz entre as plantas invasoras e a cultura (Faria et al., 1998), já que o correto manejo dessas plantas pode contribuir para manutenção das propriedades físicohídricas e mecânicas do solo, reduzir a erosão e contribuir para o aporte de matéria orgânica e ciclagem de nutrientes, o que pode resultar em acréscimo na produtividade.

O entendimento de como os diferentes sistemas de manejo das plantas invasoras afetam a capacidade de suporte de carga, bem como a resistência à compactação do solo, torna-se essencial para adaptar o manejo de lavouras cafeeiras de forma condizente. Diante dessas considerações, o objetivo deste estudo foi o de avaliar a influência dos diferentes sistemas de manejo de plantas invasoras sobre a resistência à compactação em três camadas de um Latossolo Vermelho-Amarelo (LVA), empregando modelos de capacidade de suporte de carga (CSC).

\section{MATERIAL E MÉTODOS}

O estudo foi realizado em uma área da fazenda da Epamig (Empresa de Pesquisa Agropecuária de Minas Gerais), em Patrocínio, MG, localizada na região fisiográfica do Alto Paranaíba, a uma latitude de $18^{\circ} 59$ ' 24 " S e longitude de $46^{\circ} 59$ ' 30 "W de Greenwich.
O clima da região é do tipo Aw tropical quente úmido, segundo classificação de Köppen. O clima apresenta duas estações bem definidas, sendo uma seca, que corresponde aos meses de maio a setembro, e outra chuvosa, relativa ao período de outubro a abril.

O município de Patrocínio encontra-se a uma altitude média de $934 \mathrm{~m}$, com temperatura média anual de 20 a $22{ }^{\circ} \mathrm{C}$ e precipitação média anual de $1.370 \mathrm{~mm}$; localiza-se no domínio dos planaltos e chapadas da bacia sedimentar do Paraná, na porção sudoeste do Cerrado brasileiro (Silva \& Malvino, 2005).

$\mathrm{O}$ solo da área de estudo foi classificado como Latossolo Vermelho-Amarelo (LVA) textura muito argilosa (Embrapa, 2006) (Quadro 1).

O estudo foi realizado em uma lavoura cafeeira (Coffea arabica L.) implantada em fevereiro de 1999, com a cultivar Rubi 1192 no espaçamento de $3,8 \times 0,7 \mathrm{~m}$. Foram avaliados quatro sistemas de manejo utilizados nas entrelinhas dos cafeeiros: sem capina (SC): as plantas invasoras foram deixadas em livre crescimento; capina manual (CM): as plantas invasoras foram controladas usando enxada; herbicida de pós-emergência (HPÓS): utilizou-se o ingrediente ativo glyphosate $\mathrm{N}$-(fosfonometil) glicina, na dose de $2,0 \mathrm{~L} \mathrm{ha}^{-1}$ de produto comercial e $0,72 \mathrm{~kg} \mathrm{ha}^{-1}$ de ingrediente ativo na formulação de concentrado solúvel, $360 \mathrm{~g} \mathrm{~L}^{-1}$, aplicado com volume de calda de $400 \mathrm{~L} \mathrm{ha}^{-1}$; e herbicida de pré-emergência (HPRÉ): as plantas invasoras foram controladas utilizando o ingrediente ativo oxyfluorfen (2-cloro-a,a,a-trifluorop-tolyl-3-ethoxy-4-nitrophenyl ether), na dose de $2,0 \mathrm{~kg} \mathrm{ha}^{-1}$ de produto comercial e $0,48 \mathrm{~kg} \mathrm{ha}^{-1} \mathrm{de}$ ingrediente ativo na formulação de concentrado emulsionável, $240 \mathrm{~g} \mathrm{~L}^{-1}$ (Rodrigues \& Almeida, 2005), aplicado com volume de calda de $400 \mathrm{~L} \mathrm{ha}^{-1}$.

Desde a implantação da lavoura os sistemas de manejo utilizados no centro da entrelinha foram os mesmos; o número médio de operações adotadas para controle satisfatório das plantas invasoras, durante os anos, variou de acordo com o sistema de manejo adotado. Nas condições de manejo em que se utilizou a CM foram realizadas cinco operações/ano; na

\section{Quadro 1. Caracterização física do Latossolo Vermelho-Amarelo (LVA) utilizado no experimento}

\begin{tabular}{|c|c|c|c|c|c|c|c|c|c|}
\hline Profundidade & Argila ${ }^{(1)}$ & $\operatorname{Areia}^{(1)}$ & Silte ${ }^{(1)}$ & $\mathrm{MO}^{(1,2)}$ & $\mathbf{C C}^{(1,2)}$ & $\mathbf{P M P} \mathbf{P}^{(1,2)}$ & $D s^{(3)}$ & $\mathbf{D} \mathbf{p}^{(3)}$ & $\mathbf{P} \mathbf{T}^{(3)}$ \\
\hline $\mathrm{cm}$ & 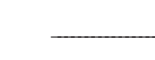 & $\mathrm{g} \mathrm{k}$ & -1 & - & $-\mathrm{kg}$ & $g^{-1}$ & $-\mathrm{M}$ & 3 & $\mathrm{~m}^{3} \mathrm{~m}^{-3}$ \\
\hline $0-3$ & 710 & 140 & 150 & 41 & 0,43 & 0,25 & 0,95 & 2,75 & 0,65 \\
\hline $10-13$ & 680 & 140 & 180 & 35 & 0,44 & 0,24 & 0,97 & 2,74 & 0,64 \\
\hline $25-28$ & 740 & 140 & 120 & 30 & 0,45 & 0,24 & 0,95 & 2,72 & 0,65 \\
\hline
\end{tabular}

\footnotetext{
(1) Média de três repetições. ${ }^{(2)}$ MO: matéria orgânica; CC: capacidade de campo-amostras submetidas ao potencial mátrico ( $\left.\Psi\right)$ de $-10 \mathrm{kPa}$; PMP: ponto de murcha permanente-amostras submetidas ao potencial mátrico $(\Psi)$ de $-1.500 \mathrm{kPa} .{ }^{(3)} \mathrm{Média}$ de 15 repetições; Ds: densidade do solo; Dp: densidade de partículas; e PT: porosidade total.
} 
aplicação do HPRÉ, duas aplicações/ano; e na de HPÓS, três aplicações/ano. Nas linhas, os cafeeiros foram mantidos limpos variando os manejos com roçacarpa, capina manual, herbicida de pós-emergência e herbicida de pré-emergência.

A amostragem foi realizada entre as datas de $12 \mathrm{a}$ 16 de julho de 2004. Nesse período, foram coletadas aleatoriamente, nas profundidades de $0-3,10-13 \mathrm{e}$ $25-28 \mathrm{~cm}, 15$ amostras indeformadas por profundidade (cinco amostras/método $\mathrm{x}$ três repetições), totalizando 180 amostras (15 amostras x 3 profundidades x 4 métodos de controle). As amostras indeformadas foram coletadas utilizando um amostrador de Uhland com anel volumétrico de $6,40 \mathrm{~cm}$ de diâmetro por $2,54 \mathrm{~cm}$ de altura. Essas profundidades de amostragem foram adotadas em razão de a maior influência dos sistemas de manejo de plantas invasoras no solo ocorrer até $30 \mathrm{~cm}$ (Alcântara \& Ferreira, 2000).

No excedente das amostras indeformadas das partes superiores e inferiores dos anéis, foram determinados os teores de matéria orgânica (Raij \& Quaggio, 1983) e densidade de partículas, pelo método do pincômetro (Blake \& Hartge, 1986b).

A capacidade de campo foi obtida em amostras indeformadas submetidas ao potencial mátrico de $(\Psi)$ -10 kPa, em mesa de tensão, e o ponto de murcha permanente $(\Psi)$, de $-1.500 \mathrm{kPa}$, em aparelho extrator de Richards (Klute, 1986).

A porosidade total foi obtida com base na densidade do solo e na densidade de partículas, pela seguinte expressão: PT = [1 - (Ds/Dp) x 100] (Vomocil, 1965).

A análise granulométrica foi feita pelo método da pipeta (Day, 1965), utilizando como dispersante químico $10 \mathrm{~mL}$ de $\mathrm{NaOH}$ a $1 \mathrm{~mol} \mathrm{~L}-1$ em contato com a amostra durante $24 \mathrm{~h}$; a dispersão mecânica foi realizada com um aparelho de ultra-som operando a 20 KHZ no estágio 9 , fornecendo potência nominal de 78,91 Watts, baseada em técnicas calorimétricas, conforme descrito por Sá et al. (2000).

As amostras indeformadas foram submetidas ao ensaio de compressão uniaxial (Bowles, 1986), modificado por Dias Junior \& Pierce (1995). As diferentes umidades das amostras, necessárias para obtenção dos modelos de CSC, foram obtidas a partir da saturação destas em bandeja com altura da lâmina de água correspondente a dois terços do cilindro. Após a saturação das amostras, o que foi obtido em média após $48 \mathrm{~h}$, elas foram secas ao ar em laboratório para obtenção das umidades. No ensaio de compressão uniaxial, as amostras indeformadas foram mantidas nos cilindros de alumínio, e estes, mantidos dentro da célula de compressão e, subseqüentemente, submetidos às pressões de 25, 50, 100, 200, 400, 800 e $1.600 \mathrm{kPa}$. Cada pressão foi aplicada até obter $90 \%$ da deformação máxima.

As amostras foram submetidas à aplicação de pressões sucessivas, sem efetuar o descarregamento das pressões previamente aplicadas (Larson \& Gupta,
1980; Kondo \& Dias Junior, 1999; Imhoff et al., 2001). Após liberação da pressão, as amostras foram secas em estufa a $105-110^{\circ} \mathrm{C}$, por $24 \mathrm{~h}$, e determinou-se a matéria seca do solo; a densidade do solo foi calculada de acordo com Blake \& Hartge (1986a). A curva de compressão foi obtida em um gráfico no qual no eixo das abscissas (X) colocou-se o logaritmo da pressão aplicada e, no das ordenadas (Y), a densidade do solo (Dias Junior \& Pierce, 1996). O valor da pressão de preconsolidação $\left(\sigma_{p}\right)$ foi calculado para cada amostra, utilizando o método proposto por Dias Junior \& Pierce (1995). As pressões de preconsolidação obtidas no ensaio de compressão uniaxial foram plotadas no eixo das ordenadas em função das umidades simuladas em laboratório, que foram plotadas no eixo das abscissas, obtendo-se os modelos de capacidade de suporte de carga ajustado de acordo com a equação $\sigma_{\mathrm{p}}=10^{(\mathrm{a}+\mathrm{bU})}$ (Dias Junior, 1994). Utilizando-se o software Sigma Plot 8.0 (2002), foram obtidas as equações matemáticas que correspondem aos modelos de capacidade de suporte de carga do solo. As comparações dos modelos foram feitas utilizando o procedimento descrito por Snedecor \& Cochran (1989), o qual testa a homogeneidade dos dados (F), e a significância dos coeficientes angular (b) e linear (a).

\section{RESULTADOS E DISCUSSÃO}

Os coeficientes dos modelos de capacidade de suporte de carga (CSC) $\left[\sigma_{\mathrm{p}}=10^{(\mathrm{a}+\mathrm{bU})}\right]$ ajustados para as camadas do LVA nas profundidades de $0-3,10-13$ e 25-28 cm, sob diferentes sistemas de manejo das plantas invasoras no centro das entrelinhas, estão apresentados nas figuras 1, 2, 3 e 4 . Os valores de "a" (intercepto da regressão linearizada) variaram de 2,66 a 2,72 , e os de "b" (coeficiente angular da regressão linearizada), entre - 0,72 e $-1,20$. Os coeficientes de determinação $\left(R^{2}\right)$ foram todos significativos a $1 \%$ pelo teste t-Student e variaram de 0,75 a 0,94 .

Para verificar as possíveis alterações da estrutura do LVA causadas pelas diferentes condições de manejo das plantas invasoras, os modelos de CSC foram comparados nas diferentes profundidades dentro de cada condição de manejo (Quadro 2).

Os modelos de CSC para o centro da entrelinha nas condições de manejo SC e HPÓS foram homogêneos para as três profundidades, segundo o teste de Snedecor $\&$ Cochran (1989). Portanto, para cada manejo, novas equações foram obtidas, considerando todos os valores de $\sigma_{\mathrm{p}} \times \mathrm{U}$ (Figuras $1 \mathrm{e} 2$ ).

Resultados semelhantes foram obtidos por Silva et al. (2006), que atribuíram a homogeneização da CSC de uma área sob Cerrado às operações de preparo do solo realizadas no desmatamento da área. Entretanto, neste estudo a homogeneização das camadas quanto à CSC deve-se também à influência do sistema radicular das plantas invasoras. 
Quadro 2. Teste de significância ${ }^{(1)}$ entre os modelos de capacidade de suporte de carga $\left[\sigma_{\mathrm{p}}=10^{(\mathrm{a}+\mathrm{bU})}\right] \mathrm{de} \mathrm{um}$ Latossolo Vermelho-Amarelo para as diferentes condições de manejo das plantas invasoras e camadas no centro das entrelinhas

\begin{tabular}{|c|c|c|c|c|}
\hline \multirow{2}{*}{$\begin{array}{l}\text { Sistema de manejo das } \\
\text { plantas invasoras }\end{array}$} & \multirow{2}{*}{ Camada $(\mathrm{cm})$} & \multirow{2}{*}{$\mathbf{F}$} & \multicolumn{2}{|c|}{$\mathbf{F}$} \\
\hline & & & Coeficiente angular, b & Coeficiente linear, a \\
\hline \multirow[t]{2}{*}{ Sem Capina (SC) } & $0-3$ vs $10-13$ & $\mathrm{H}$ & $\mathrm{ns}$ & $\mathrm{ns}$ \\
\hline & $0-3$ e $10-13$ vs $25-28$ & $\mathrm{H}$ & $\mathrm{ns}$ & $\mathrm{ns}$ \\
\hline \multirow{2}{*}{$\begin{array}{l}\text { Herbicida de Pós- } \\
\text { emergência (HPÓs) }\end{array}$} & $0-3$ vs $10-13$ & $\mathrm{H}$ & $\mathrm{ns}$ & $\mathrm{ns}$ \\
\hline & $0-3$ e $10-13$ vs $25-28$ & $\mathrm{H}$ & $\mathrm{ns}$ & $\mathrm{ns}$ \\
\hline \multirow[t]{3}{*}{ Capina Manual (CM) } & $0-3$ vs $10-13$ & $\mathrm{NH}$ & $\mathrm{ns}$ & $* *$ \\
\hline & $0-3$ vs $25-28$ & $\mathrm{H}$ & $\mathrm{ns}$ & $\mathrm{ns}$ \\
\hline & $0-3$ e $25-28$ vs $10-13$ & $\mathrm{NH}$ & $\mathrm{ns}$ & $\mathrm{ns}$ \\
\hline \multirow{3}{*}{$\begin{array}{l}\text { Herbicida de Pré- } \\
\text { emergência (HPRÉ) }\end{array}$} & $0-3$ vs $10-13$ & $\mathrm{H}$ & $* *$ & $\mathrm{~ns}$ \\
\hline & $0-3$ e $10-13$ vs $25-28$ & $\mathrm{H}$ & $* *$ & $* *$ \\
\hline & $10-13$ vs $25-28$ & $\mathrm{H}$ & $\mathrm{ns}$ & $* *$ \\
\hline
\end{tabular}

(1) Snedecor \& Cochran (1989). H: homogêneo; NH: não-homogêneo; **: significativo a 1 \%; ns: não-significativo.

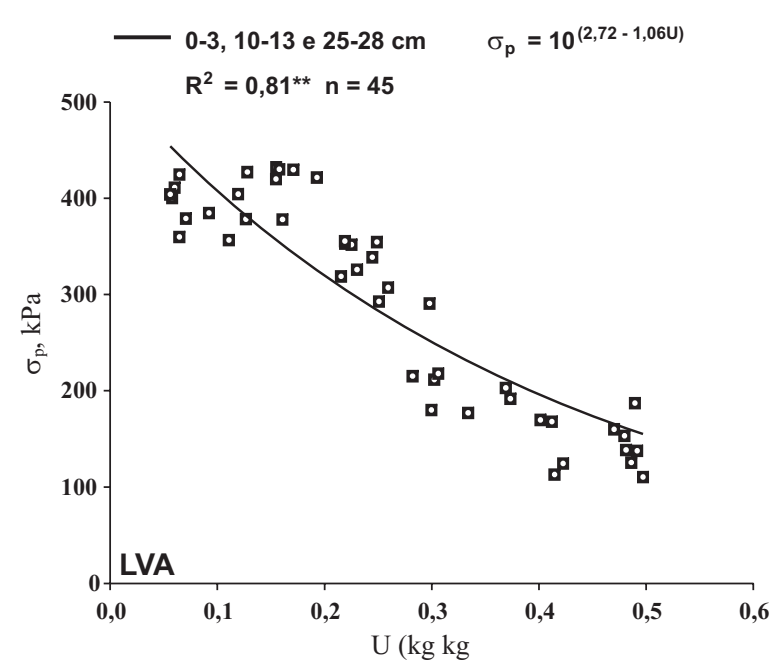

Figura 1. Modelo de capacidade de suporte de carga (CSC) para o sistema de manejo sem capina (SC) no centro da entrelinha, para as camadas de 0-3, 10-13 e 25-28 cm. U: umidade, $\sigma_{\mathrm{p}}$ : pressão de preconsolidação.

Na condição de manejo HPÓS ocorre morte total das plantas invasoras, permanecendo o sistema radicular no solo, que, após sua decomposição, forma canalículos, os quais favorecem a infiltração e redistribuição de água e trocas gasosas, bem como os ciclos de umedecimento e secagem em profundidade, que promovem a homogeneização da CSC da estrutura do solo. Além disso, a cobertura morta proporcionada pelo manejo com HPÓS e a cobertura das plantas invasoras no manejo SC protegem o solo contra erosão e conservam a sua umidade, contribuindo para sustentabilidade do sistema. Com isso, pode-se indicar

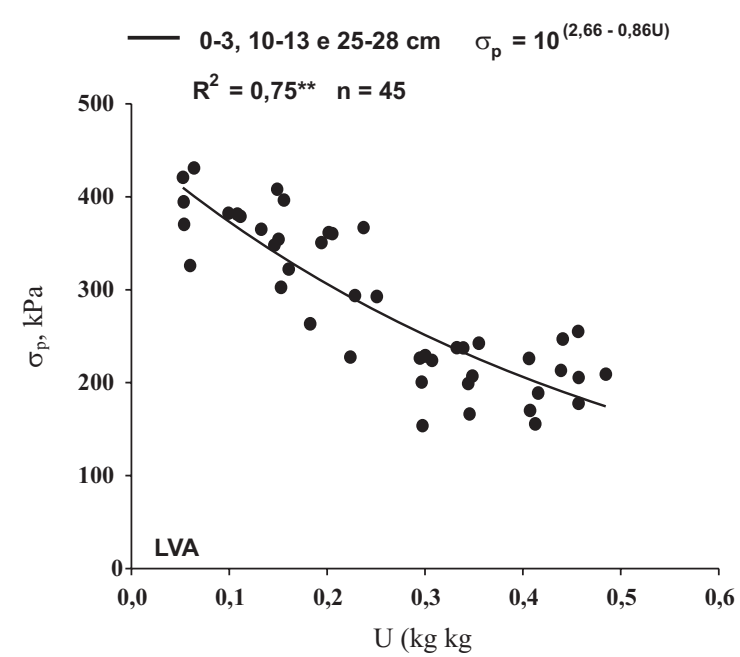

Figura 2. Modelo de capacidade de suporte de carga (CSC) para o sistema de manejo herbicida de pósemergência (HPÓS) no centro da entrelinha, para as camadas de 0-3, 10-13 e $25-28 \mathrm{~cm}$. U: umidade, $\sigma_{\mathrm{p}}$ : pressão de preconsolidação.

o uso dos manejos HPÓS e SC, que proporcionam boa cobertura vegetal na entrelinha do cafeeiro, com o objetivo de reduzir a degradação física e as perdas de água e solo (Faria et al., 1998; Bertoni \& Lombardi Neto, 1999; Prochnow et al., 2005), além de reduzirem a remoção de partículas com nutrientes e defensivos agrícolas. Semelhantemente ao que ocorre nas áreas sob cultivos conservacionistas, como o plantio direto e cultivo mínimo (Assis \& Lanças, 2005; Singh \& Malhi, 2006), os resíduos das plantas invasoras podem aumentar a deposição de matéria orgânica e melhorar a agregação do solo. Esses fatores podem contribuir 
para melhoria das propriedades físicas e promover homogeneização das camadas do solo quanto à CSC (Figuras 1 e 2).

Os modelos de CSC do solo, para o centro da entrelinha na condição de manejo $\mathrm{CM}$, foram homogêneos para as camadas de 0-3 e $25-28 \mathrm{~cm}$ (Quadro 2), sendo, portanto, ajustada uma nova equação considerando todos os valores de $\sigma_{p}$ e U (Figura 3). Na camada de 10-13 cm, os modelos de CSC foram estatisticamente diferentes daqueles das camadas de $0-3$ e $25-28 \mathrm{~cm}$. Observou-se que a camada de 10-13 cm apresentou maior CSC em qualquer condição de umidade do que as camadas de 0-3 e 25-28 cm (Figura 3), mostrando-se mais resistente à compactação, o que pode limitar o crescimento do sistema radicular da cultura nesta condição de manejo. Esse comportamento pode ser explicado pela maior resistência mecânica natural desta camada, resultante dos processos pedogenéticos.

Na condição de manejo de plantas invasoras onde não existe influência do sistema radicular, como HPRÉ, o comportamento das camadas quanto à CSC foi diferente (Quadro 2 e Figura 4). A camada de 0-3 cm apresentou maior CSC para umidades superiores a $0,20 \mathrm{~kg} \mathrm{~kg}^{-1}$, demonstrando ser esta camada mais resistente à compactação do solo em umidades elevadas, quando comparada com as de 10-13 e 25-28 cm. Esse resultado pode ser atribuído à formação do selamento superficial proporcionado pelo efeito indireto da utilização constante do HPRÉ.

Portanto, a utilização constante do HPRÉ e, conseqüentemente, a exposição do solo ao impacto das gotas da chuva potencializam o selamento superficial do solo (Faria et al., 1998; Bertoni \& Lombardi Neto, 1999; Alcântara \& Ferreira, 2000). O selamento
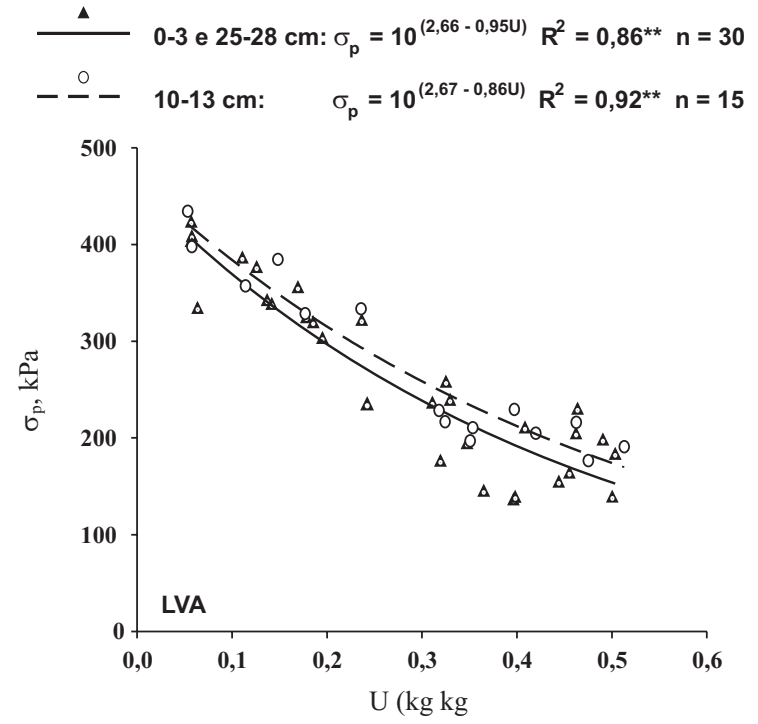

Figura 3. Modelos de capacidade de suporte de carga (CSC) para o sistema de manejo capina manual (CM) no centro da entrelinha, para as camadas de 0-3, 25-28 e 10-13 cm. U: umidade, $\sigma_{\mathrm{p}}$ : pressão de preconsolidação.

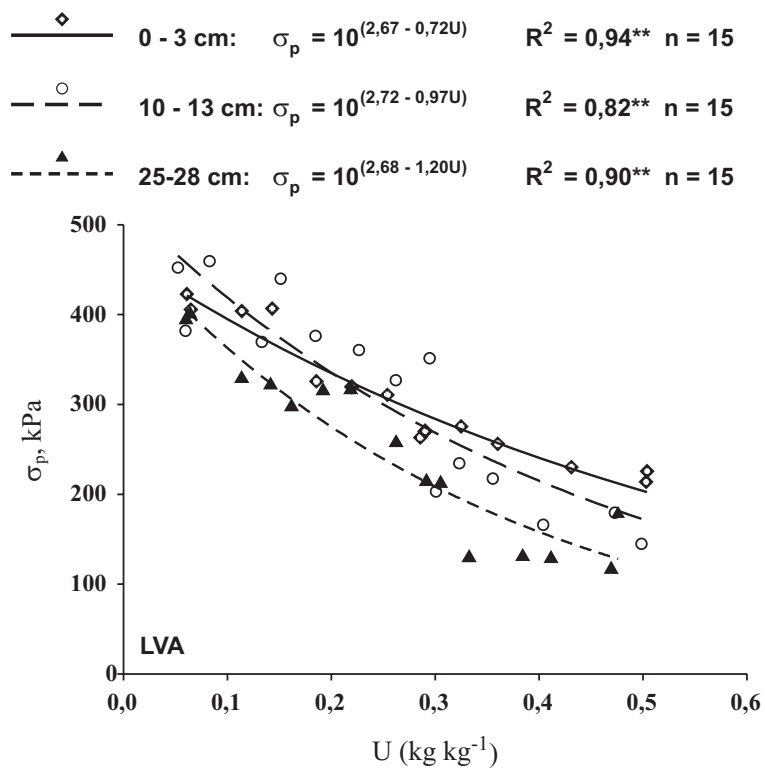

Figura 4. Modelos de capacidade de suporte de carga (CSC) para o sistema de manejo herbicida de pré-emergência (HPRÉ) no centro da entrelinha, para as camadas de 0-3, 10-13 e 25-28 cm. U: umidade, $\sigma_{\mathrm{p}}$ : pressão de preconsolidação.

superficial caracteriza-se por elevada densidade do solo, pequena porosidade e baixa condutividade hidráulica (Silva \& Kato, 1997), fatores estes que interferem no comportamento compressivo do solo e, por conseguinte, na pressão de preconsolidação, refletindo em maior CSC.

Adicionalmente, o sistema de manejo HPRÉ expõe o solo, tornando-o mais suscetível aos agentes erosivos; conseqüentemente, a desestruturação e perda da capacidade de absorção de água resultante do entupimento dos poros provoca maior escoamento superficial, que, por sua vez, intensifica a erosão (Faria et al., 1998; Alcântara \& Ferreira, 2000). Adicionalmente, o impacto direto das gotas de chuva e dos ciclos de umedecimento e secagem, bem como a ação do herbicida como agente desorganizador da estrutura, provoca rearranjamento das partículas, proporcionando o aparecimento de camadas impermeáveis à água, devido à migração das partículas de argila, que obstrui os poros.

A formação do selamento superficial em áreas manejadas constantemente com herbicidas de préemergência foi observada por Faria et al. (1998) e, em lavouras cafeeiras, por Alcântara \& Ferreira (2000).

Em lavouras cafeeiras, o aumento da intensidade das operações mecanizadas ocorre na estação chuvosa (Silva et al., 2006), em que o solo apresenta alta umidade, o que aumenta o potencial de provocar dano à sua estrutura (Alakukku et al., 2003; Dias Junior et al., 2005). Com elevada CSC no centro das entrelinhas, o LVA manejado com HPRE torna-se menos suscetível à compactação, na camada de 0 $3 \mathrm{~cm}$ (Figura 4); contudo, maior CSC do solo resultará 
em maior resistência mecânica imposta ao sistema radicular da cultura (Imhoff et al., 2001). Solos com grande resistência mecânica podem ser benéficos à trafegabilidade de máquinas para o manejo da lavoura cafeeira, porém podem afetar severamente a expansão do sistema radicular e o transporte de água e de assimilados das raízes para a parte aérea (Rena \& Guimarães, 2000). Já a camada de $25-28 \mathrm{~cm}$ apresentou menor CSC para qualquer condição de umidade (Figura 4), sendo, portanto, a mais suscetível à compactação e menos impeditiva ao crescimento do sistema radicular.

Outro aspecto que vale ressaltar é que, em virtude do entupimento dos poros ocasionado pelo selamento superficial na camada de $0-3 \mathrm{~cm}$, as camadas mais profundas mantêm-se constantemente úmidas e menos sujeitas aos ciclos de umedecimento e secagem, tornando mais uniforme a umidade e aliviando, assim, a resistência mecânica natural do solo (Miranda et al., 2003; Silva et al., 2006). Maior umidade com menores oscilações após picos de precipitação, nas camadas profundas de um solo manejado com herbicida de pré-emergência, foi observada por Faria et al. (1998).

Para verificar possíveis alterações na CSC do LVA no centro das entrelinhas, provocadas pelos diferentes sistemas de manejo das plantas invasoras, os modelos de CSC foram comparados (Quadro 3).

Quando o controle das plantas invasoras foi realizado no centro da entrelinha de plantio, utilizando os sistemas de manejo SC e HPÓS, os modelos de CSC foram semelhantes em todas as camadas avaliadas, e estes, homogêneos aos sistemas de manejo CM nas camadas de $0-3,25-28 \mathrm{~cm}$ e HPRÉ na camada de 10-13 cm (Quadro 3). Portanto, uma nova equação foi ajustada, considerando todos os valores de $\sigma_{\mathrm{p}} \mathrm{e} U$ obtendo-se um único modelo de CSC, expresso pela equação $\sigma_{\mathrm{p}}=10^{(2,69-0,97 \mathrm{U})}, \mathrm{R}^{2}=0,79 * * \mathrm{e} n=135$ (Figura 5).

Os sistemas de manejos CM na camada de 10 $13 \mathrm{~cm}$ e HPRÉ nas camadas de 0-3 e 25-28 cm não foram homogêneos (Quadro 3), indicando diferentes CSC. Esses resultados indicam a influência desses sistemas de manejo das plantas invasoras no arranjo das unidades estruturais ao longo das profundidades (Figura 5). A maior CSC para o sistema de manejo HPRÉ na camada de $0-3 \mathrm{~cm}$ foi observada em umidades superiores a $0,10 \mathrm{~kg} \mathrm{~kg}^{-1}$, sendo este sistema o mais resistente à compactação na superfície. Já a camada de 25-28 cm para o sistema de manejo HPRÉ apresentou menor CSC, quando comparado com qualquer outra condição de manejo em todas as faixas

Quadro 3. Teste de significância ${ }^{(1)}$, entre os modelos de capacidade de suporte de carga $\left[\sigma_{p}=10^{(a+b U)}\right]$ de um Latossolo Vermelho-Amarelo para diferentes sistemas de manejo de plantas invasoras e camadas no centro das entrelinhas

\begin{tabular}{|c|c|c|c|}
\hline \multirow{2}{*}{ Sistema de manejo das plantas invasoras } & \multirow{2}{*}{$\mathbf{F}$} & \multicolumn{2}{|c|}{$\mathbf{F}$} \\
\hline & & Coeficiente angular, b & Coeficiente linear, a \\
\hline $\begin{array}{l}\mathrm{SC}(0-3,10-13,25-28 \mathrm{~cm}) \text { vs HPÓs }(0-3,10-13 \mathrm{e} \\
25-28 \mathrm{~cm})\end{array}$ & $\mathrm{H}$ & ns & $\mathrm{ns}$ \\
\hline $\begin{array}{l}\mathrm{SC}(0-3,10-13,25-28 \mathrm{~cm}) \text { e HPÓs }(0-3,10-13 \mathrm{e} \\
25-28 \mathrm{~cm}) \text { vs CM }(0-3 \text { e } 25-28 \mathrm{~cm})\end{array}$ & $\mathrm{H}$ & $\mathrm{ns}$ & $\mathrm{ns}$ \\
\hline $\begin{array}{l}\mathrm{SC}(0-3,10-13,25-28 \mathrm{~cm}) \text { e HPÓs }(0-3,10-13 \mathrm{e} \\
25-28 \mathrm{~cm}) \text { e CM }(0-3 \text { e } 25-28 \mathrm{~cm}) \text { vs HPRÉ }(10-13 \mathrm{~cm})\end{array}$ & $\mathrm{H}$ & $\mathrm{ns}$ & $\mathrm{ns}$ \\
\hline $\begin{array}{l}\mathrm{SC}(0-3,10-13,25-28 \mathrm{~cm}) \text { e HPÓs }(0-3,10-13 \mathrm{e} \\
25-28 \mathrm{~cm}) \text { e CM }(0-3 \text { e } 25-28 \mathrm{~cm}) \text { vs HPRÉ }(0-3 \mathrm{~cm})\end{array}$ & $\mathrm{NH}$ & $* *$ & * \\
\hline $\begin{array}{l}\mathrm{SC}(0-3,10-13,25-28 \mathrm{~cm}) \text { e HPÓS }(0-3,10-13 \mathrm{e} \\
25-28 \mathrm{~cm}) \text { e CM }(0-3 \text { e } 25-28 \mathrm{~cm}) \text { e HPRÉ }(10-13 \mathrm{~cm}) \\
\text { vs HPRÉ }(25-28 \mathrm{~cm})\end{array}$ & $\mathrm{H}$ & $\mathrm{ns}$ & ** \\
\hline CM $(10-13 \mathrm{~cm})$ vs HPRÉ $(0-3 \mathrm{~cm})$ & $\mathrm{H}$ & $\mathrm{ns}$ & ** \\
\hline CM $(10-13 \mathrm{~cm})$ vs HPRÉ $(25-28 \mathrm{~cm})$ & $\mathrm{NH}$ & * & $* *$ \\
\hline
\end{tabular}


de umidade (Figura 5), resultando com isso em maior suscetibilidade à compactação do solo em subsuperfície. Portanto, lavouras manejadas com HPRÉ apresentam menor CSC na camada de $25-28 \mathrm{~cm} \mathrm{e}$ podem ser menos restritivas ao crescimento do sistema radicular da cultura. Entretanto, o tráfego de máquinas deve ser aplicado com ressalvas, visto que em maiores profundidades é difícil a recuperação dos efeitos nocivos da compactação do solo. Em um Latossolo Amarelo distrófico no município de Patrocínio, MG, Silva et al. (2006) concluíram que operações realizadas com a colhedora KTR, pulverizador Arbus 2000, roçadora e efeito cumulativo do tráfego de todos estes equipamentos, na época seca (solo com menor umidade e maior CSC), ainda assim, provocaram compactação nas camadas de 0-3 e 25$28 \mathrm{~cm}$. O sistema de manejo CM na camada de 10 $13 \mathrm{~cm}$ mostrou comportamento intermediário em relação a CSC, quando comparado ao manejo HPRÉ nas camadas de $0-3$ e $25-28 \mathrm{~cm}$.

De maneira geral, a CSC decresce no centro da entrelinha na seguinte ordem: HPRÉ na camada de $0-3 \mathrm{~cm}>$ CM na camada de $10-13 \mathrm{~cm}>$ SC nas camadas de $0-3,10-13$ e $25-28 \mathrm{~cm}=$ HPÓS nas camadas de $0-3,10-13$ e 25-28 cm = CM nas camadas de $0-3$ e $25-28 \mathrm{~cm}=$ HPRÉ na camada de $10-13 \mathrm{~cm}$ $>$ HPRÉ na camada de $25-28 \mathrm{~cm}$.

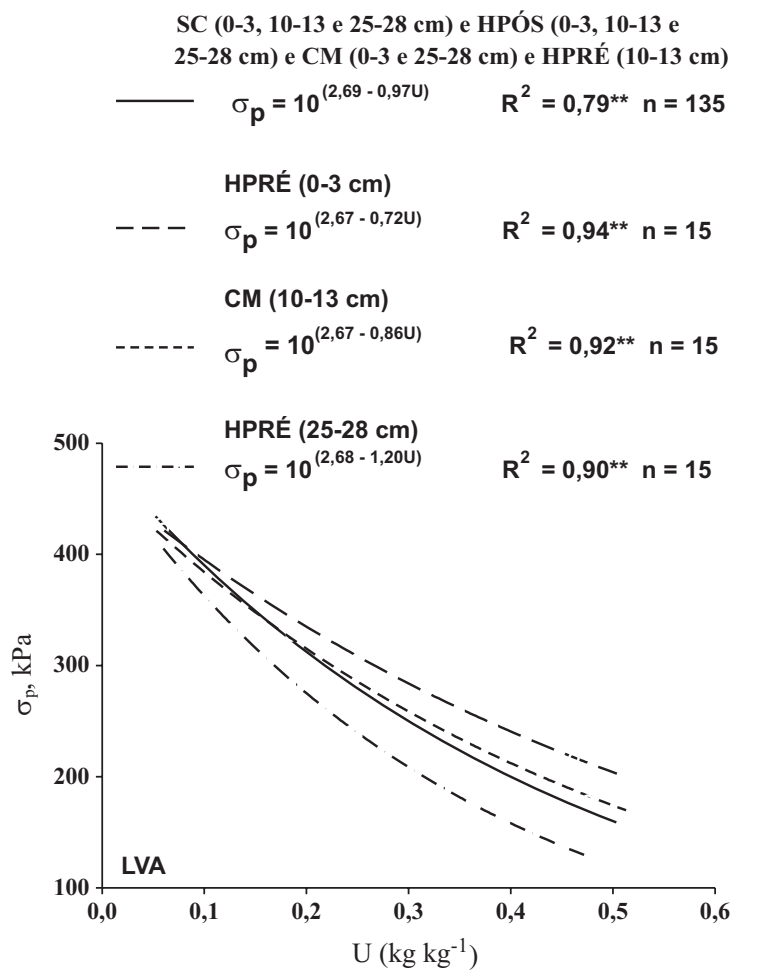

Figura 5. Modelos de capacidade de suporte de carga (CSC) para o centro da entrelinha, para as camadas de 0-3, 10-13 e 25-28 cm, submetidos a diferentes sistemas de manejo das plantas invasoras. $U$ : umidade, $\sigma_{\mathrm{p}}$ : pressão de preconsolidação.

\section{CONCLUSÕES}

1. A camada que se mostrou mais suscetível à compactação foi a de $25-28 \mathrm{~cm}$ do sistema de manejo HPRE, e a mais resistente, a camada de $0-3 \mathrm{~cm}$ deste mesmo sistema de manejo.

2. Os sistemas de manejo SC e HPÓS nas camadas de $0-3,10-13$ e $25-28 \mathrm{~cm}, \mathrm{CM}$ nas camadas de $0-3$ e 25-28 cm e HPRÉ na camada de $10-13 \mathrm{~cm}$ apresentaram a mesma resistência à compactação.

\section{AGRADECIMENTOS}

À CAPES e ao CNPq, pela concessão das bolsas de estudo aos alunos de pós-graduação. Ao Consórcio Brasileiro de Pesquisa e Desenvolvimento do Café (CBP\&D/Café), pelo auxílio financeiro para execução do trabalho. Ao Pesquisador da EPAMIG/CTSM, Dr. Elifas Nunes de Alcântara, por conceder a área do estudo e pelo auxílio no desenvolvimento do trabalho.

\section{LITERATURA CITADA}

AGRIANUAL, 2006. FNP Consultoria e Comércio. Anuário da agricultura brasileira. São Paulo, 2006. 504p.

ALAKUKKU, L.; WEISSKOPF.; CHAMEN, W.C.T.; TIJINK, F.G.J.; van der LINDEN, J.P.; PIRES, S.; SOMMER, C. \& SPOOR, G. Prevention strategies for field trafficinduced subsoil compaction: A review. Part I Machine/soil interactions. Soil Till. Res., 73:145-160, 2003.

ALCÂNTARA, E.N. \& FERREIRA, M.M. Efeito de métodos de controle de plantas invasoras na cultura do cafeeiro (Coffea arabica L.) sobre a qualidade física do solo. R. Bras. Ci. Solo, 24:711-721, 2000.

ASSIS, R.L. \& LANÇAS, K.P. Avaliação da compressibilidade de um Nitossolo Vermelho distroférrico sob sistema plantio direto, preparo convencional e mata nativa. $\mathrm{R}$. Bras. Ci. Solo, 29:507-514, 2005.

ASSOCIAÇÃO BRASILEIRA DE CAFÉS ESPECIAIS - BSCA. Lista de verificação sistemas de gestão sócio-ambiental. Anexo RA 0552.04 ver. 01. 2005.

BERTONI, J. \& LOMBARDI NETO, F. Conservação do solo. 4.ed. São Paulo, Ícone, 1999. 355p.

BLAKE, G.R. \& HARTGE, K.H. Bulk density. In: KLUTE, A., ed. Methods of soil analysis. Madison, Soil Science Society of America-American Society of Agronomy, 1986a. Part 1. p.363-375.

BLAKE, G.R. \& HARTGE, K.H. Particle density. In: KLUTE, A., ed. Methods of soil analysis. 2.ed. Madison, Soil Science Society of America-American Society of Agronomy, 1986b. Part 1. p.377-382. (Agronomy Monograph, 9) 
BOWLES, J.E. Engineering properties of soils and their measurements. 3.ed. New York, McGraw-Hill, 1986. $218 p$.

CANILLAS, E.C. \& SALOKHE, V.M. A decision support system for compaction assessment in agricultural soils. Soil Till. Res., 65:221-230, 2002.

DAY, P.R. Particle fractionation and particle-size analysis. In: BLACK, C.A., ed. Methods of soil analysis. Madison, American Society of Agronomy, 1965. Part I. p.545-567. (Agronomy Monograph, 9)

DIAS JUNIOR, M.S. Compression of three soils under long-term tillage and wheel traffic. East Lansing Michigan, State University, 1994. 114p. (Tese de Doutorado)

DIAS JUNIOR, M.S.; FONSECA, S.; ARAUJO JUNIOR, C.F. \& SILVA, A.R. Soil compaction due to forest harvest operations. Pesq. Agropec. Bras., 42:257-264, 2007.

DIAS JUNIOR, M.S.; LEITE, F.P.; LASMAR JUNIOR, E. \& ARAUJO JUNIOR, C.F. Traffic effects on the soil preconsolidation pressure due to Eucalyptus harvest operations. Sci. Agric., 62:248-255, 2005.

DIAS JUNIOR, M.S. \& PIERCE, F.J. Revisão de literatura. O processo de compactação do solo e sua modelagem. R. Bras. Ci. Solo, 20:175-182, 1996.

DIAS JUNIOR, M. S. \& PIERCE, F.J. A simple procedure for estimating preconsolidation pressure from soil compression curves. Soil Technol., 8:139-151, 1995.

EMPRESA BRASILEIRA DE PESQUISA AGROPECUÁRIA - EMBRAPA. Centro Nacional de Pesquisas de Solos. Sistema brasileiro de classificação de solos. 2.ed. Rio de Janeiro, Embrapa Solos, Brasília, Sistema de Produção de Informação, 2006. 306p.

FARIA, J.C.; SHAEFER, C.E.R.; RUIZ, H.A. \& COSTA, L.M. Effects of weed control on physical and micropedological properties of Brazilian Ultisol. R. Bras. Ci. Solo, 22:731-741, 1998.

IMHOFF, S.; SILVA, A.P.; DIAS JUNIOR, M.S. \& TORMENA, C.A. Quantificação de pressões críticas para o crescimento das plantas. R. Bras. Ci. Solo, 25:1118,2001

KLUTE, A. Laboratory measurement of hydraulic conductivity of satured soils. Madison, American Society of Agronomy, 1986. p.253-261. (Agronomy Monograph, 9)

KONDO, M.K. \& DIAS JUNIOR, M.S. Compressibilidade de três Latossolos em função da umidade e uso. R. Bras. Ci. Solo, 23:211-218, 1999.

LARSON, W.E. \& GUPTA, S.C. Estimating critical stress in unsaturated soils from changes in pore water pressure during confined compression. Soil Sci. Soc. Am. J., 44:1127-1132, 1980.
MIRANDA, E.E.V.; DIAS JUNIOR, M.S.; GUIMARÃES, P.T.G.; PINTO, J.A.O.; ARAUJO JUNIOR, C.F. \& LASMAR JUNIOR, E. Efeito do manejo e do tráfego nos modelos de sustentabilidade da estrutura de um Latossolo Vermelho cultivado com cafeeiros. Ci. Agrotec., Edição Especial:1506-1515, 2003.

PENG, S.H.; HORN, R.; ZHANG, B. \& ZAHO, Q.G. Mechanisms of soil vulnerability to compaction of homogenized and recompacted Ultisols. Soil Till. Res., 76:125-137, 2004.

PROCHNOW, D.; DECHEN, S.C.F.; DE MARIA, I.C. \& CASTRO, O.M. Razão de perdas de terra e fator C da cultura do cafeeiro em cinco espaçamentos, em Pindorama (SP). R. Bras. Ci. Solo, 29:91-98, 2005.

RAIJ, B. van \& QUAGGIO, J.A. Métodos de análises de solo para fins de fertilidade. Campinas, Instituto Agronômico de Campinas, 1983. 21p. (Boletim Técnico, 81)

RENA, A.B. \& GUIMARÃES, P.T.G. Sistema radicular do cafeeiro: Estrutura, distribuição, atividades e fatores que o influenciam. Belo Horizonte, EPAMIG, 2000. 80p. (Série Documentos, 37)

RODRIGUES, B.N. \& ALMEIDA, F.S. Guia de herbicidas. 5.ed. Londrina, 2005. 592p.

SÁ, M.A.C.; LIMA, J.M. \& LAGE, G. Procedimento padrão para medida da potência liberada pelo aparelho ultrasom. Comunicação. Ci. Agrotec., 24:300-306, 2000.

SIGMA PLOT, Scientific Graphing Software. Versão 8.0, San Rafael, Jandel Corporation, 2002.

SILVA, A.R.; DIAS JUNIOR, M.S.; GUIMARÃES, P.T.G. \& ARAUJO JUNIOR, C.F. Modelagem da capacidade de suporte de carga e quantificação dos efeitos das operações mecanizadas em um Latossolo Amarelo cultivado com cafeeiros. R. Bras. Ci. Solo, 30:207-216, 2006.

SILVA, C.L. \& \& KATO, E. Efeito de selamento superficial na condutividade hidráulica saturada da superfície de um solo sob Cerrado. Pesq. Agropec. Bras., 32:213$220,1997$.

SILVA, E.M. \& MALVINO, S.S.A.B. Análise climática do município de Patrocínio (MG). Caminhos Geografia, 10:93-108, 2005. Disponível em: <www. ig. ugu. br/revista/ caminhos. html>

SILVA, R.B.; DIAS JUNIOR, M.S.; SILVA, F.A.M. \& FOLE, S.M. O tráfego de máquinas agrícolas e as propriedades físicas, hídricas e mecânicas de um Latossolo dos Cerrados R. Bras. Ci. Solo, 27:211-218, 2003.

SINGH, B. \& MALHI, S.S. Response of soil physical properties to tillage and residue management on two soils in a cool temperate environment. Soil Till. Res., 85:143-53, 2006 
SNEDECOR, G.W. \& COCHRAN, W.G. Statical methods. 8.ed. Ames, State University Press, 1989. 503p.
VOMOCIL, J.A. Porosity. In: BLAKE, C.A., ed. Methods of soil analysis. Madison, American Society of Agronomy, 1965. 